\title{
Endoscopic Ultrasonography Fine Needle Aspiration in Pancreatic Masses: A Study Comparing 22-gauge and 25-gauge Needle in the Same Patient
}

\author{
Vasseur $\mathbf{P}^{1,2}$, Puppa $\mathbf{G}^{3}$, Nguyen $\mathbf{T}^{1}$, Genevay $\mathbf{M}^{3}$ and Frossard $\mathrm{JL}^{1 *}$ \\ ${ }^{1}$ Service of Gastroenterology and Hepatology, Geneva University Hospital, Rue Gabrielle-Perret-Gentil 4, 1205 Genève, Switzerland \\ ${ }^{2}$ Service of Gastroenterology, CHU Poitiers, 2 Rue de la Milétrie, 86021 Poitiers, France \\ ${ }^{3}$ Service of Clinical Pathology, Geneva University Hospital, Rue Gabrielle-Perret-Gentil 4, 1205 Genève, Switzerland \\ "Corresponding author: Frossard JL, Service of Gastroenterology and Hepatology, Geneva University Hospital, Rue Gabrielle-Perret-Gentil 4, 1205 Genève, \\ Switzerland, Tel: 41223729340; Fax: 41223729366; E-mail: jean-louis.frossard@hcuge.ch
}

Received date: Nov 18, 2015; Accepted date: Dec 26, 2015; Published date: Dec 28, 2015

Copyright: (c) 2015 Frossard JL, et al. This is an open-access article distributed under the terms of the Creative Commons Attribution License, which permits unrestricted use, distribution and reproduction in any medium, provided the original author and source are credited.

\begin{abstract}
Background: It remains unclear whether the 22- and 25-gauge needles have equal diagnostic yields in endoscopic ultrasonography-fine needle aspiration of pancreatic masses.

Methods: Patients presenting with pancreatic solid masses were prospectively included between July 2010 and June 2012. Each patient underwent ultrasonography-fine needle aspiration using two different needle sizes (22- and 25-gauge needles) during the same endoscopic session, the 25 and 22 needle sequence being randomized. Cytological preparations included smear cytology, ThinPrep and Cell Block. Specimens were analyzed for diagnosis, cellularity, amount of blood and digestive contamination. Final diagnosis was reached by the final pathological report, clinical and radiological follow-up.
\end{abstract}

Results: Thirty-seven patients were included among them 34 subjects had malignant pancreatic neoplasm on final diagnosis. Pancreatic masses were located in the head of pancreas in $73 \%$ of cases. The mean size of the lesions was $34.6 \pm 14.4 \mathrm{~mm}$. Diagnostic yields of the 25- and 22-gauge needles were $85.3 \%(95 \% \mathrm{Cl}$ : $72.6-98)$ and $88.2 \%(95 \% \mathrm{Cl}: 76.7-99.7)$ respectively $(\mathrm{p}>0.05)$. Diagnosis was obtained with smear cytology alone in $58.8 \%$ patients with the 22 -gauge needle and in $55.9 \%$ patients with the 25 -gauge needle, in $64.5 \%$ patients with Cell Block for both needles and in $76.5 \%$ with ThinPrep for both needles. The quality of specimens was comparable with the two needles. No complication occurred.

Conclusion: The 25- and 22-gauge needles carry the same diagnostic yield when performing ultrasonographyfine needle aspiration of solid pancreatic masses. ISRCTN69545565 Controlled trials.

Keywords: Endoscopic ultrasonography; Fine needle aspiration; Pancreatic neoplasms

\section{Introduction}

Fine-needle aspiration guided by endoscopic ultrasonography (EUS-FNA) has changed the management of solid pancreatic masses. While EUS can provide supplementary information regarding the staging of pancreatic tumors, FNA can be safely performed on pancreatic masses and/or peripancreatic lymph nodes to bring the ultimate diagnosis of malignancy. Because the differential diagnosis of solid pancreatic masses is wide including benign, premalignant and malignant conditions, sampling the mass is of great interest before sending the patient to demanding and complicated surgery. However, tumor sampling is currently recommended only when the pancreatic mass does not seem amenable to resection because of staging results or poor physical condition. In case of potentially resectable tumor, EUSFNA is usually performed in order to exclude unusual tumors that would not benefit from surgery such as lymphoma [1]. EUS-FNA has a high accuracy in diagnosing malignant pancreatic tumors. A recent meta-analysis including almost 5'000 patients who underwent EUS-
FNA indicates a pooled sensitivity of $85 \%$ when considering malignant cytology [2]. Of the three needle sizes available to puncture solid pancreatic masses (19-gauge, 22-gauge or 25-gauge), the 22-gauge needle is the most widely used device although there is no clear recommendation to choose among them. It is indeed unclear whether the 25-gauge needle, which is the thinner one, could provide equal or better performance in sampling the pancreas than the 22-gauge needle. It is also unclear whether the 25-gauge needle can decrease the risk of FNA-induced bleeding and gastro-intestinal contamination in the smears and cell block. In prospective studies comparing the results brought by EUS FNA using a 22 -gauge and a 25 -gauge needles for pancreatic masses, the 25-gauge needle appeared to have the same diagnostic yield than the 22-gauge needle [3,4]. However, the design of these studies did not compare the results obtained by the two needle types in the same patient, but compared the respective yield of each needle used in two different groups of patients randomly assigned for either a 25-gauge puncture or a 22-gauge puncture. The validity of these trials is also limited by the low number of patients enrolled [5-7]. The aim of this study was to compare, in a larger cohort, the diagnostic accuracy of a 25-gauge and a 22-gauge needles in the same patient presenting with pancreatic solid mass. 
Citation: Vasseur P, Puppa G, Nguyen T, Genevay M, Frossard JL (2016) Endoscopic Ultrasonography Fine Needle Aspiration in Pancreatic Masses: A Study Comparing 22-gauge and 25-gauge Needle in the Same Patient. J Cytol Histol 7: 384. doi: $10.4172 / 2157-7099.1000384$

Page 2 of 4

\section{Patients and Methods}

\section{Trial design and intervention}

The study is a randomized, prospective, non-inferiority study aimed at evaluating the diagnostic yield of EUS FNA using both 25- and 22gauge needle in the same patient. The protocol was approved and cleared by our local Ethical Committee. The reporting of the trial was based on the CONSORT 2010 recommendations. The study was conducted at the Geneva university Hospital and was given number ISRCTN69545565 from Controlledtrials.com (www.controlledtrials.com).

EUS FNA was performed using two needle, a 25-gauge and a 22gauge needle, in the same patient. The randomization sequence of the needle size was created with a 1:1 allocation using blocks of 4 without stratification. The allocation was achieved in the operating room by physicians blinded for the allocation sequence, using sequentially numbered, sealed and opaque envelopes. The corresponding envelope was opened only after the eligible participant had signed an informed consent form explaining all the modalities of the endoscopic procedure. Data collection was performed blindly by a noninterventional gastroenterologist.

\section{EUS FNA procedure}

EUS FNA was performed by three endosonographers (JLF, JMD and TNG). Patients were sedated using propofol in a left lateral position. A linear echoendoscope (EUM 160, Olympus, Hamburg, Germany) was used for FNA, doppler analysis was performed to avoid any intervened vessel in the path of the needle. Three passages without stylet in place were performed with each needle in the solid pancreatic mass, a trangastric approach being used for body and tail lesions while a transduodenal approach was used for lesions in the head and uncinate process of the pancreas. A $10 \mathrm{cc}$ succion was used for each passage. The needle was rinsed with $0.5 \mathrm{ml}$ of $\mathrm{NaCl} 0.9 \%$. The collected specimen from each needle were first smeared and rinsed for Cell Block and ThinPrep preparations.

Complications associated with FNA were defined as GI bleeding by either mouth or anus, abdominal pain, acute pancreatitis and fever every item occurring within 24 hours after the procedure.

\section{Cytopathology assessment and final diagnosis}

Specimens obtained with the two needles were blindly reviewed by 2 experienced cytopathologists (MG, GP). The material that was separately evaluated included: thin-Prep, cell block and cytological smear. For each case slide labels indicated the type of needle used (22 or 25) thereby a distinct assessment was performed.

Every slide was scanned at low magnification and a semiquantitative assessment was applied allowing a 4-tiered classification, as follows:

$0=$ Absence; $1=$ Very rare cells; $2=$ Few cells aggregates and a few isolated cells;

3 = Several cells aggregates, up to covering almost all the slide.

These criteria were applied for evaluating the amount of: a) Tumor cells, b) Gastro-intestinal contamination and c) Red blood cells.

The final diagnosis was based on cytology report, surgical pathology if available, repeated radiological imaging and clinical follow-up.

\section{Sample size, inclusions and exclusions criteria}

The sample size calculation was based on previous studies comparing the two explored strategies (diagnostic yield of 25- and 22gauge needle size) demonstrating a diagnostic yield of $85 \%$ in each arm. In order to detect the same diagnostic yield afforded by each needle size, when requiring a two-sided alpha $=0.05$ and a statistical power of 0.8 , a sample size of 35 patients was estimated as the minimum number as each patient was his/her own control. Thirty seven patients referred to our Hospital for EUS-FNA of solid pancreatic masses were prospectively enrolled between July 2010 and June 2012. Written informed consent was obtained before their participation in the study.

Only adults $>18$ years were eligible for the study. They all presented to our service with a solid pancreatic mass identified by at least two dissimilar imaging modalities (ultrasound, CT scan, MRI). Pancreatic mass was defined as abnormal nodule located within any segment of the pancreas.

Exclusion criteria were sepsis, acute pancreatitis defined as abdominal pain associated with increased serum lipase $>3$ the normal value), anticoagulant therapy, anti-agregant therapy other than aspirin, previous history of modified anatomy interfering with endoscopic assessment and incapacity to give informed consent.

\section{Statistical analysis}

Statistical analysis was performed using SAS version 9.3 (SAS Inc, Cary, NC, USA). Cochran-Mantel-Haenszel test was used for categorical variables (diagnostic yield), Wilcoxon signed ranks test was used for ordinal variables (contamination, amount of blood and cellularity). Results were considered statistically significant if $\mathrm{P}$ value $<0.05$. The minimum sample size was determined on the basis of a non-inferiority of the 25-gauge needle for diagnostic yield, admitting a loss of $0.4 \%$ in sensitivity.

\section{Results}

Between July 2010 and June 2012, 73 patients were assessed for eligibility. Forty-five patients did not meet the inclusion criteria. A total of 37 patients with pancreatic solid mass was prospectively enrolled. There were 21 men and 16 women with a mean age of $69.3 \pm$ 12 years (Table 1). Pancreatic masses were mainly located $(73 \%)$ in the head or in the uncinate process of the pancreas, the mean size of the lesions being $34.6 \pm 14.4 \mathrm{~mm}$ (Table 1). Among the 37 patients included, 3 patients had a final diagnosis of non-malignant mass according to surgical reports, clinical follow-up ( $>18$ month) and imaging studies. Inflammatory masses were related to either chronic pancreatitis in 2 patients and to acute pancreatitis in one case (Figure $1)$. In the 34 patients with a final diagnosis of malignant tumor, the majority of lesions (82\%) were pancreatic carcinomas. Diagnosis was obtained by EUS-FNA in 32 patients (94\%), whereas surgical biopsy was mandatory in the 2 remaining patients. None of the patients presented hemorrhagic or infectious complications after EUS-FNA.

\section{Cytological diagnosis}

When considering the 34 patients with a final diagnosis of malignant tumor, diagnostic yield of 22-gauge and 25-gauge needles was significantly non-different $(\mathrm{p}=0.37)$. Diagnosis was made in 30 patients (88.2\%; 95\%CI: 76.7-99.7) with the 22-gauge needle and in 29 subjects (85.3\%; 95\%CI: 72.6-98) with the 25 -gauge needle. According 
Citation: Vasseur P, Puppa G, Nguyen T, Genevay M, Frossard JL (2016) Endoscopic Ultrasonography Fine Needle Aspiration in Pancreatic Masses: A Study Comparing 22-gauge and 25-gauge Needle in the Same Patient. J Cytol Histol 7: 384. doi: 10.4172/2157-7099.1000384

Page 3 of 4

to the type of cytological preparation (smear, ThinPrep or Cell Block) the diagnostic yield of both needles was not different $(\mathrm{p}>0.05)$.

\begin{tabular}{|l|l|}
\hline Characteristics of population & \\
\hline Median age(y, range) & $72(42-87)$ \\
\hline Gender (males, \%) & $21(56.8)$ \\
\hline Site of lesion (n, \%) & \\
\hline Head / uncinate & $27(73)$ \\
\hline Body / tail & $10(27)$ \\
\hline Median size of lesion (mm, range) & $30.5(17-82)$ \\
\hline
\end{tabular}

Table 1: Baseline characteristics

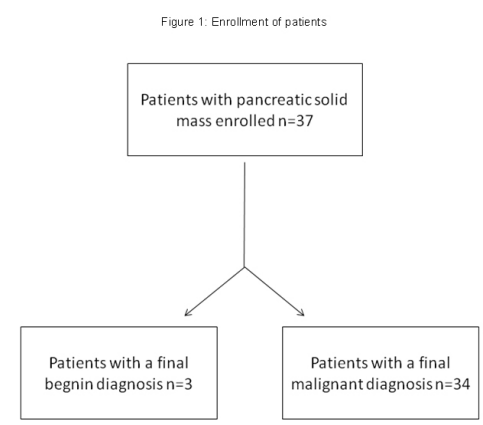

Figure 1: Enrollment of patients

Diagnosis was obtained with smear cytology alone in $58.8 \%$ patients with the 22 -gauge needle and in $55.9 \%$ patients with the 25 -gauge needle, in $64.5 \%$ patients with Cell Block for both needles and in $76.5 \%$ with ThinPrep for both needles (Table 2).

\begin{tabular}{|l|l|l|l|}
\hline & 22-gauge & 25-gauge & \\
\hline Overall diagnosis & & & * \\
\hline Yes (n, \%) & $30(88.2)$ & $29(85.3)$ & \\
\hline No (n, \%) & $4(11.8)$ & $5(14.7)$ & \\
\hline Diagnosis with smear cytology & & & \\
\hline Yes (n, \%) & $20(58.8)$ & $19(55.9)$ & \\
\hline No (n, \%) & $14(41.2)$ & $14(44.1)$ & \\
\hline Diagnosis with Thin-Prep & & & \\
\hline Yes (n, \%) & $26(76.5)$ & $26(76.5)$ & \\
\hline No (n, \%) & $8(23.5)$ & $8(23.5)$ & \\
\hline Diagnosis with Cell Block & & $11(35.5)$ & \\
\hline Yes (n, \%) & $20(60.4)$ & $20(60.4)$ & \\
\hline No (n, \%) & $11(35.5)$ & 1 & \\
\hline
\end{tabular}

Table 2: Diagnostic yield of the 22-gauge and 25-gauge needles

\section{Cytological results}

Smear cytology and ThinPrep were available in the 34 patients with a diagnosis a malignant tumor, Cell Block was available in 30 patients. Quality of samples did not differ between the two sizes of needles. Cellularity of samples, regardless of cytological preparation, was not inferior with 25-gauge needles. Furthermore, the contamination by digestive cells and the amount of blood was not different for the two needles (Table 3).

\begin{tabular}{|l|l|l|l|l|l|l|l|l|l|}
\hline & \multicolumn{2}{|l|}{$\begin{array}{l}\text { Smear } \\
\text { cytology }\end{array}$} & \multicolumn{3}{|l|}{ Thin-Prep } & \multicolumn{3}{|l|}{ Cell Block } & \\
\hline & $\begin{array}{l}\mathbf{2 2} \\
\text { G }\end{array}$ & $\mathbf{2 5 G}$ & $\mathbf{p}$ & $\mathbf{2 2 G}$ & $\mathbf{2 5 G}$ & $\mathbf{p}$ & $\mathbf{2 2 G}$ & $\mathbf{2 5 G}$ & $\mathbf{p}$ \\
\hline $\begin{array}{l}\text { Amount of } \\
\text { blood (n) }\end{array}$ & & & 0.51 & & & 1.0 & & & 1.0 \\
\hline $\mathbf{0}$ & 11 & 9 & & 9 & 8 & & 10 & 11 & \\
\hline $\mathbf{1}$ & 10 & 9 & & 15 & 16 & & 12 & 11 & \\
\hline $\mathbf{2}$ & 4 & 7 & & 9 & 9 & & 4 & 7 & \\
\hline $\mathbf{3}$ & 9 & 9 & & 1 & 1 & & 4 & 2 & \\
\hline $\begin{array}{l}\text { Contamination } \\
\text { (n) }\end{array}$ & & & 0.90 & & & 0.15 & & & 0.78 \\
\hline $\mathbf{0}$ & 6 & 7 & & 6 & 10 & & 10 & 11 & \\
\hline $\mathbf{1}$ & 13 & 12 & & 16 & 15 & & 17 & 15 & \\
\hline $\mathbf{2}$ & 10 & 7 & & 8 & 6 & & 3 & 4 & \\
\hline $\mathbf{3}$ & 5 & 8 & & 4 & 3 & & 0 & 1 & \\
\hline Cellularity (n) & & & 0.52 & & & 0.12 & & & 0.98 \\
\hline $\mathbf{0}$ & 11 & 14 & & 7 & 9 & & 10 & 9 & \\
\hline $\mathbf{1}$ & 11 & 9 & & 10 & 13 & & 9 & 10 & \\
\hline $\mathbf{2}$ & 7 & 6 & & 11 & 9 & & 2 & 8 & \\
\hline $\mathbf{3}$ & 5 & 5 & & 6 & 3 & & 9 & 4 & \\
\hline
\end{tabular}

Table 3: Cytological results of samples obtained with the 22-gauge and 25-gauge needles

\section{Discussion}

EUS-FNA has become a standard in pancreatic masses diagnosis and much work has been done to improve its diagnostic accuracy in the recent years. Although the 19-gauge needle and Trucut biopsy needles allow obtaining larger specimens, they carry a higher risk of bleeding and are less flexible in the trans-duodenal approach than thinner needles [8]. These features explain the reason why thinner needles either the 22-gauge or the 25-gauge needles are used instead for pancreatic masses. The latter may be in theory associated with a lower incidence of FNA-induced complications. In a retrospective study including 842 patients with pancreatic masses, no complication were noted in the 25-gauge needle group [9]. Moreover, this needle had a higher technical success rate and was easier to manipulate, especially in lesions of the pancreas located in the head and the uncinate process $[3,5]$. Nevertheless, it is still unclear whether the 25and 22-gauge needles have the same diagnostic yield. Three randomized trials compared the diagnostic yield afforded by the two 
Citation: Vasseur P, Puppa G, Nguyen T, Genevay M, Frossard JL (2016) Endoscopic Ultrasonography Fine Needle Aspiration in Pancreatic Masses: A Study Comparing 22-gauge and 25-gauge Needle in the Same Patient. J Cytol Histol 7: 384. doi: 10.4172/2157-7099.1000384

Page 4 of 4

needles in patients with pancreatic masses and did not show any difference $[3,4,10]$. However, the full interpretation of these studies is somewhat difficult as they did not compare the accuracy of the two needles in the same lesions and furthermore in the same patient. Two pilot studies including 9 and 24 patients respectively compared the 22and 25-gauge needles in the same patient and demonstrated both accurate diagnosis in these small groups of patients $[5,6]$. A third study collecting 50 patients lead to the same result with a diagnostic accuracy reaching $94 \%$ and $86 \%$ for the 25 - and 22 -gauge needles respectively [7]. We also show that both needles without the stylet in place have equal diagnostic yields for pancreatic masses, reaching $85 \%$ for the 25 gaugne needle and $88 \%$ for the 22 -gauge needle. Depending on the type of cytological preparation, the size of the needle did not influence diagnostic accuracy. Combining the three cytological preparation increased the final accuracy, but we interestingly observed that ThinPrep showed a trend towards higher diagnostic yield than smear cytology ( $76 \%$ vs. $59 \%, \mathrm{p}>0.05)$; This characteristic is somewhat in contradiction with the findings of Leblanc et al. who showed that sensitivity of on-site smear cytology for pancreatic cancer was $98 \%$ versus $62 \%$ for ThinPrep [11]. We also show that the quality of cytological specimens was comparable with the two needles, which was consistent with previous studies [6,7]. The small number of patients included in this study may represent a limitation but could be explained by its particular design since it was a non-inferiority study. Nevertheless, to the best of our knowledge, this is the only study that studied the performance of two different needles in the same patient.

\section{References}

1. Dumonceau JM, Polkowski M, Larghi A, Vilmann P, Giovannini M, et al (2011) Indications, results, and clinical impact of endoscopic ultrasound (EUS)-guided sampling in gastroenterology. European Society of Gastrointestinal Endoscopy (ESGE) Clinical Guideline, Endoscopy 43: 897-912.

2. Hewitt MJ, McPhail MJW, Possamai L, Dhar A, Vlavianos P, et al. (2012) EUS-guided FNA for diagnosis of solid pancreatic neoplasms: A metaanalysis. Gastrointestinal endoscopy 75: 319-31.
3. Lee JK, Lee KT, Choi ER, Jang TH, Jang KT, et al. (2013) A prospective, randomized trial comparing 25-gauge and 22-gauge needles for endoscopic ultrasound-guided fine needle aspiration of pancreatic masses. Scandinavian Journal of Gastroenterology 48: 752-7.

4. Camellini L, Carlinfante G, Azzolini F, Iori V, Cavina M, et al. (2011) A randomized clinical trial comparing $22 \mathrm{G}$ and $25 \mathrm{G}$ needles in endoscopic ultrasound-guided fine-needle aspiration of solid lesions. Endoscopy 43: 709-15.

5. Sakamoto H, Kitano M, Komaki T, Noda K, Chikugo T, et al. (2009) Prospective comparative study of the EUS guided 25-gauge FNA needle with the 19-gauge Trucut needle and 22-gauge FNA needle in patients with solid pancreatic masses. Journal of Gastroenterology and Hepatology 24: 384-90.

6. Lee JH, Stewart J, Ross WA, Anandasabapathy S, Xiao L, et al. (2009) Blinded prospective comparison of the performance of 22-gauge and 25 gauge needles in endoscopic ultrasound-guided fine needle aspiration of the pancreas and peri-pancreatic lesions. Digestive diseases and sciences 54: 2274-81.

7. Fabbri C, Polifemo AM, Luigiano CV, Cennamo V, Baccarini P, et al. (2011) Endoscopic ultrasound-guided fine needle aspiration with 22- and 25-gauge needles in solid pancreatic masses: A prospective comparative study with randomisation of needle sequence. Digestive and liver disease 43: 647-52.

8. Harris MD, Buscaglia JM (2010) How to do pancreatic mass FNA. Gastrointestinal endoscopy 71: 825-6.

9. Yusuf TE, Ho S, Pavey DA, Michael H, Gress FG (2009) Retrospective analysis of the utility of endoscopic ultrasound-guided fine-needle aspiration (EUS-FNA) in pancreatic masses, using a 22-gauge or 25gauge needle system: A multicenter experience. Endoscopy 41: 445-8.

10. Siddiqui UD, Rossi F, Rosenthal LS, Padda MS, Murali-Dharan V, et al. (2009) EUS-guided FNA of solid pancreatic masses: A prospective, randomized trial comparing 22-gauge and 25-gauge needles. Gastrointestinal endoscopy 70: 1093-7.

11. LeBlanc JK, Emerson RE, Dewitt J, Symms M, Cramer HM, et al. (2009) A prospective study comparing rapid assessment of smears and ThinPrep for endoscopic ultrasound-guided fine-needle aspirates. Endoscopy 42: 389-94. 